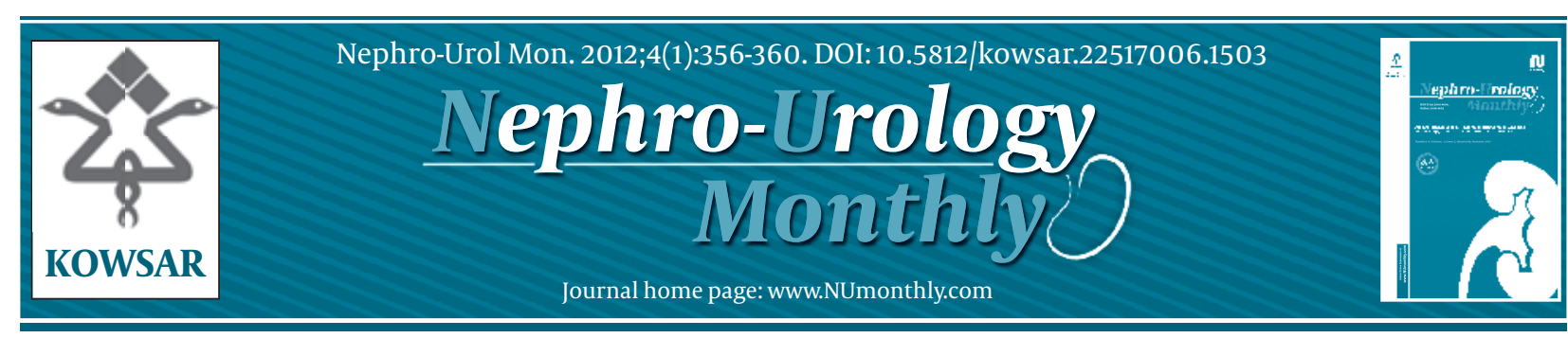

\title{
Case Creation and E-Learning in a Web-Based Virtual Department of Urology Using the INMEDEA Simulator
}

\author{
Marcus Horstmann ${ }^{1,2^{*}}$, Carolin Horstmann ${ }^{3}$, Markus Renninger ${ }^{1}$ \\ ${ }^{1}$ Department of Urology, Eberhard Karls University Tuebingen, Tuebingen, Germany \\ ${ }^{2}$ Department of Urology, Kantonsspital Winterthur, Winterthur, Switzerland \\ ${ }^{3}$ Department of Psychiatry, IPW Winterthur, Winterthur, Switzerland
}

\begin{tabular}{l}
\hline A R T I C L E I N F O \\
\hline Article type: \\
Original Article \\
\hline Article history: \\
Received: 29 May 2011 \\
Revised: 13 Jun 2011 \\
Accepted: 25 Jun 2011 \\
\hline
\end{tabular}

Keywords:

Learning

Internet

Urology Department, Hospital

\begin{abstract}
A B S T R A C T
Background: Web based e-learning with virtual patient cases has become a valuable tool in medical education.

Objectives: We report our experiences with the INMEDEA Simulator as both teachers and case authors in the subspecialty of urology.

Materials and Methods: The INMEDEA Simulator is a fully graphical interactive web application of a virtual hospital. Users can work on virtual patients online in various specialty clinics, including a department of urology. Each step, from acquiring a patient's medical history to conducting a physical examination and further diagnostic tests, can be performed on the simulator to solve the virtual cases. An expert system gives feedback on best medical practices. For medical teachers, an authoring tool is integrated into the platform. In the department of urology, virtual patient cases were used and evaluated in a blended teaching approach.

Results: Fourteen virtual patient cases representing the most common urological diseases are currently available in the urology department. Cases can be solved in different languages. When virtual simulation was integrated into a blended teaching approach, students' acceptance rates were high. Virtual patient simulation has been described as a good addition to classical medical teaching, but not as a substitute for traditional instruction. From the teachers' point of view, the simulator offers an excellent opportunity to present clinical problems and information to students. New clinical cases and content can be easily generated and rapidly integrated using the authoring tool.

Conclusions: Web based e-learning with virtual patient cases on the INMEDEA Simulator offers an excellent opportunity to improve practical knowledge on the basis of problem solving. Case creation by using the authoring tool is easy.
\end{abstract}

Copyright $\odot 2012$, Kowsar M.P.Co. All rights reserved.

- Implication for health policy/practice/research/medical education:

With continuing advances in computer and internet technology web based e-learning with virtual patients becomes an option even for undergraduate medical students. We present our experience with the INMEDEA Simultor in the subspecialty of urology.

- Please cite this paper as:

Horstmann M, Horstmann C, Renninger M. Case Creation and E-Learning in a Web-Based Virtual Department of Urology Using the INMEDEA Simulator.Nephro-Urol Mon.2012;4(1):356-60. DOI:10.5812/kowsar.22517006.1503

\section{Background}

Recent developments in computer technology and the current widespread availability of high-speed internet

\footnotetext{
* Corresponding author: Marcus Horstmann, Kantonsspital Winterthur, Department of Urology, Brauerstr. 15, 8401 Winterthur, Switzerland. Tel: +41-522664171, Fax:+41-522664503, E-mail: marcushorstmann@gmx.ch

DOI:10.5812/kowsar.22517006.1503

Copyright $\odot 2012$, Kowsar M.P.Co. All rights reserved.
}

have improved the options for e-learning in medical education $(1,2)$. With the introduction of handheld computers, smart phones, and smaller laptops, many medical students and teachers have gained additional wireless access to the world wide web and use it to enhance their medical studies (3). Many universities now offer online services that facilitate administrative processes or provide access to libraries and other medical content $(4,5)$. 
Beyond these services, current technology also allows teaching programs to utilize even more complex webbased learning platforms $(6,7)$, such as the INMEDEA Simulator with its interactive patient simulation (8-10).

\section{Objectives}

Since problem-solving-based teaching has recently attracted more attention from medical teachers, we report our e-learning experiences, both as authors and teachers, using the INMEDEA Simulator in the urology subspecialty.

\section{Materials and Methods}

\subsection{INMEDEA Simulator}

The INMEDEA Simulator is an interactive e-learning management system built around a virtual hospital that can be accessed after individual online password registration at http://www.inmedea-simulator.net. The platform hosts fully graphical and multimedial illustrations created by a single designer. By mouseover for menu selection, users can enter the virtual hospital and navigate freely around the virtual campus to visit various departments, which include almost all medical subspecialties. There are 25 subspecialties currently available, including departments of urology, surgery, medicine, gynecology, pediatrics, neurology, ophthalmology, dermatology, and more.

In this simulation of a real hospital, virtual patients can be seen for several visits in each department depending on their medical problem. During the simulation, the user has to act as the medical doctor in charge of the patient, finding and resolving the patient's medical problem. First, the student must select a patient and lead the patient into the virtual room of a specific clinic (Figure 1). Then, the user takes the medical history of the patient by using the mouse to select sequential menu items and "asks" standardized questions. Answers by the patient are provided as text statements. Next, the user has to perform a physical examination, with findings obtained either in a written format or by multimedia presentations that illustrate the case. The student then orders laboratory, radiological, endoscopic, or functional tests to arrive at the correct diagnosis for the patient's condition. Finally, the user must offer a treatment. Again, the results of each test are displayed in both written and multimedia formats.

The aim of the simulation is for the student to arrive at the right diagnosis in a reasonable amount of time by using the most appropriate diagnostic steps in order to save time and money. During the simulation, an integrated expert system gives feedback on best medical practices and resource consumption. Additional links within the case encourage further reading in a virtual library or allow chatting with colleagues in a virtual café. All of this content is written and edited by case authors who are chosen as experts in their respective fields. At the end of the simulation, both the accuracy of the diagnosis and the adequacy of the diagnostic steps are electronically evaluated by the computer software. This content is also written and added by the case author during case creation. All performance results can be viewed electronically by the user at the end of each case.

Currently, the INMEDEA Simulator is owned by INMEDEA GmbH Company, which independently cooperates with medical schools, online services, and medical publishers who contract for its services. This project, originally called "Prometheus" was started at the University of Tuebingen, Germany and was initially supported by the German Ministry of Education and Science. After winning the Medida Prix, an award for digital media in scientific education, in 2005, the project was taken over by INMEDEA GmbH Company in 2006. During this time, more than 60 case authors have created, written, and illustrated more than 250 medical cases, which are now available in the virtual hospital. The platform was initially created in the German language and later translated into several other languages, mostly European. Currently, the simulator can be used in 11 different languages, including English, Spanish, Italian, Greek, and French.

\subsection{The INMEDEA Authoring Tool}

For authors, the INMEDEA Simulator offers an easy-touse tool to constantly improve and add new cases to the simulator. This authoring tool is integrated into the INMEDEA Simulator and is also accessible after online registration. To create a new virtual patient, the author first writes a storyboard that describes the patient, including his appearance and medical history. The patient is then graphically designed and given a virtual name, and his characteristics are entered into the authoring tool. Next, the numbers of visits are determined, and the author has to limit the amount of questions, laboratory investigations, and other possible diagnostic steps that will be available to the user. Following the storyboard, the author then prepares written answers to each standardized question (Figure 2). All answers are typed and edited in templates that also allow the user to accept appropriate standardized answers provided by the simulator via a simple mouse click. This authoring tool facilitates the easy creation of new cases. The same tools are available for establishing all other results of further medical examinations and diagnostic tests to be displayed in written form. Again, if results are "normal," the authors can rely on templates that offer standardized "normal" results for investigations that are routinely conducted. All added content is stored on the server and can be used directly in the simulator. Consequently, the author can constantly review all results of his work, and an additional preview option facilitates control of the editing process. During case creation, the author not only chooses and writes the questions and patients' answers but also writes and edits expert comments, which can be further used as expert information by the user when working on a case. To further illustrate each case, multimedia data files are uploaded and automatically integrated into the system. At the end of the authoring process, the author 
has to select a main diagnosis for each visit and must determine the most appropriate treatment for his patient. Then, cases are summarized in an assessment that includes a discussion about treatment alternatives. Most cases were first written in German and later translated into other languages. Editing is possible in all available languages.

\section{Results}

Between 2002 and 2007, 14 virtual urological patient cases of common urological problems and diseases were created for the virtual urology department of the INMEDEA Simulator. Currently, the virtual urology department includes 11 male and 3 female patients, and these patients represent the most common urological problems and diseases. Four patients have prostate cancer (2 local diseases, 2 advanced diseases), 2 have benign prostrate hyperplasia (BPH), 2 have testicular pain or mass, and 1 each has flank pain, advanced renal cell carcinoma, hematuria, and repeated urinary infection. Recently, other authors have added 2 andrological cases to the department as well.

All authors have found that case creation using the integrated authoring tool, as presented above, is easy to use and complete. At the beginning of the project's launch, all data first had to be typed into separate excel files and were then stored locally; however, the present authoring tool offers an internet-based platform that directly saves all data on a web server. This gives authors the opportunity to work online freely at any place and any time. It also allows several authors to work simultaneously on a case. For example, the integrated review system permits supervising authors to raise queries and add their corrections. All multimedia files for case illustrations are uploaded easily into the system, including large video files. Cases can be constantly corrected, updated, revised, or changed. This process is still in place today. While at the beginning of the project's launch, case creation took several weeks, cases can now be created within 10-20 working hours.

We evaluated the INMEDEA Simulator in medical school courses in 2007 and 2008 with seventy $4^{\text {th }}$-year medical students at the University of Tuebingen. Students were asked to solve 2 patient cases prior to the beginning of their urology courses. Cases were later discussed and solved together with a medical teacher during course sections with 6-8 students in a blended teaching approach. In this setting, all online learning was accompanied by classical medical teaching in small groups by a medical teacher. All students were given full access to all urological cases during the respective year. Teaching was evaluated after the completion of the course using an 18-item questionnaire based on Likert scales. Results showed that this teaching approach was well accepted and appreciated by the students. Seventy-six percent of the students judged the course as good or very good, and $87 \%$ felt that the course offered a way to better understand urological diseases. Seventy-two percent of the students stated that they had fun solving the cases. In free text statements, students also stated that case-based learning with virtual patients was useful for their medical education; however, virtual learning should only be viewed as a preparation for their courses and not as a substitute for contact with real patients, which is still considered indispensible. In this evaluation of the simulator in 2007 to 2008 , students did report that several technical problems still limited the simulator and that the system was often too slow to access medical content. These problems have now been completely resolved after careful consideration of student comments and by using further technological advances.

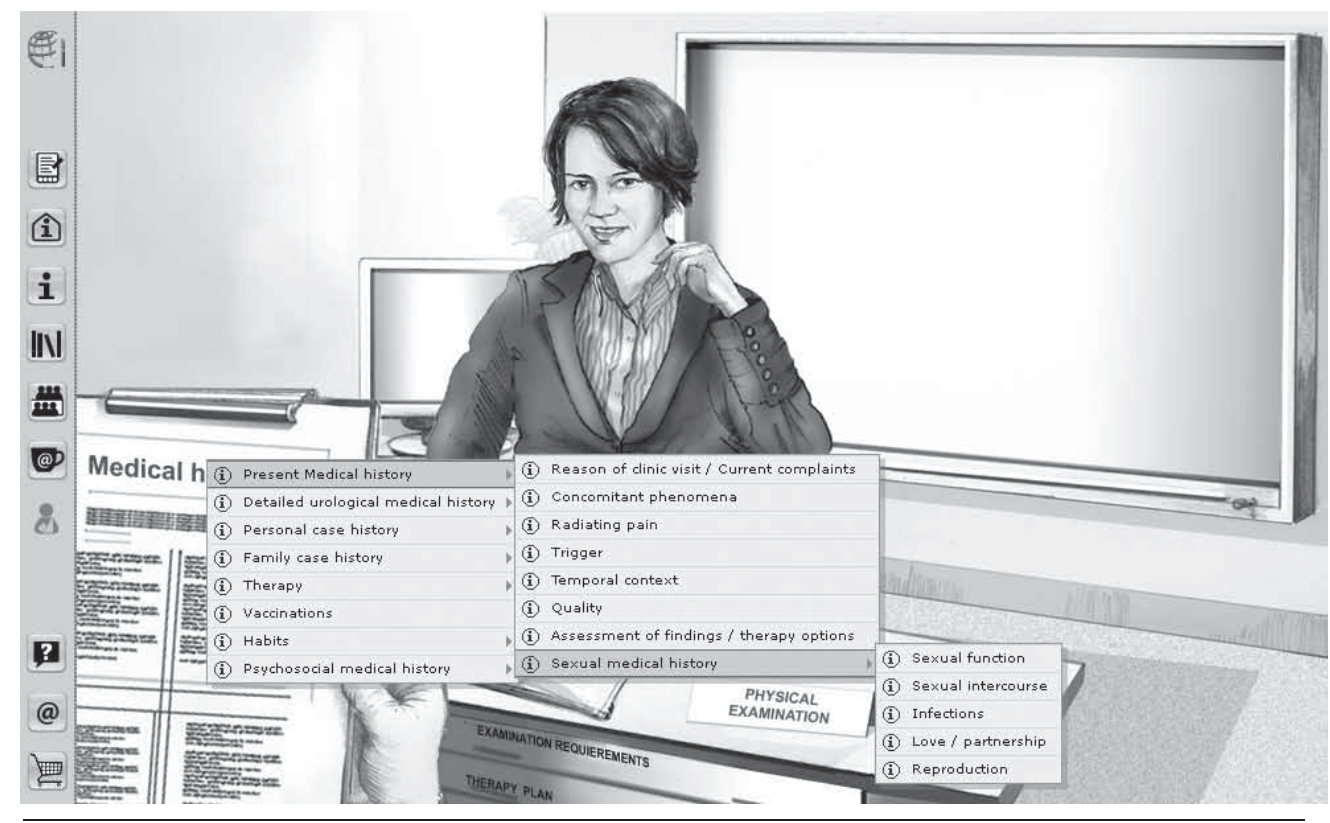

Figure 1. Screenshot: Taking a Patient's Medical History by Mouseover Selection 


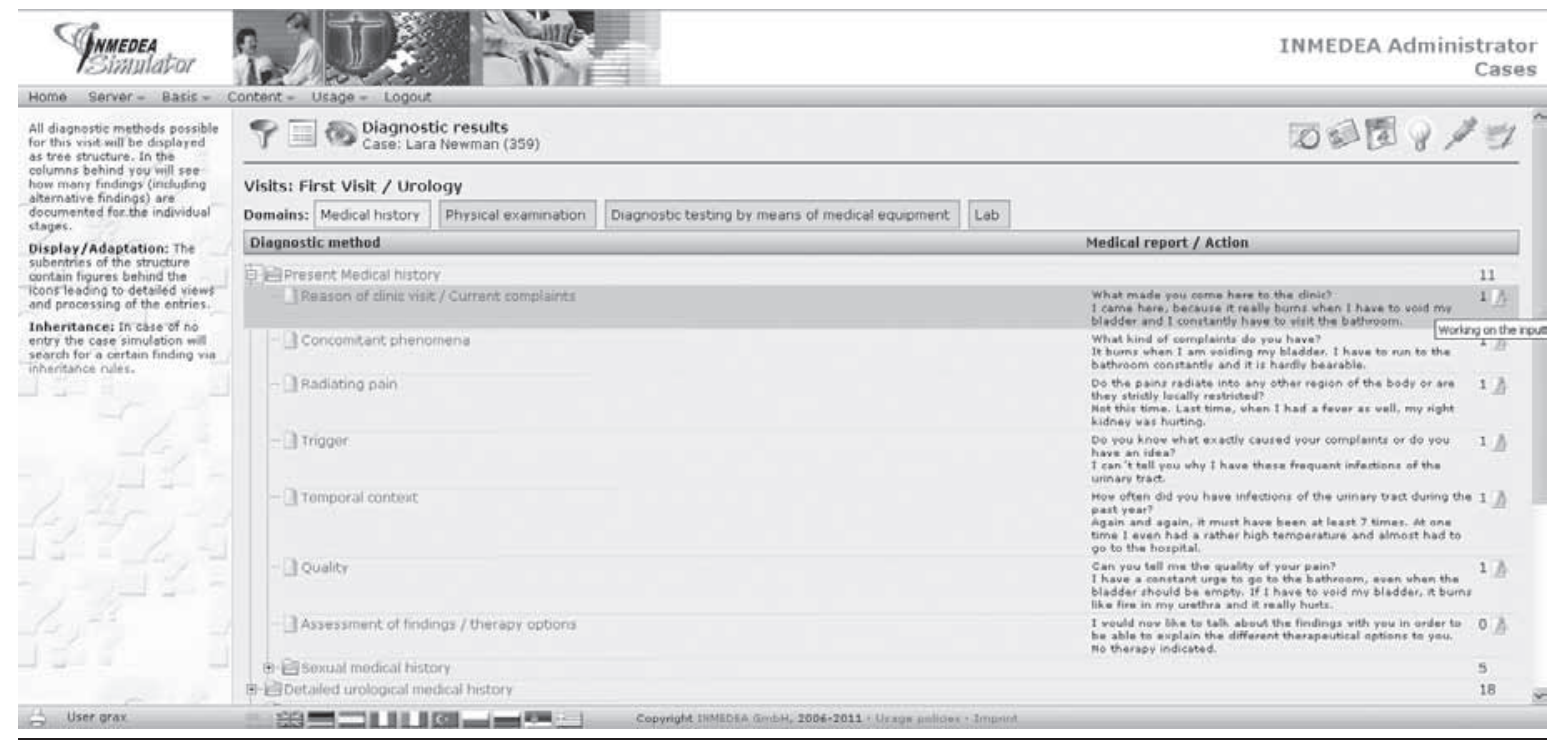

Figure 2. Screenshot: Structure of the Medical History Report in the Authoring Tool (Case: Lara Newman During 1st Visit)

\section{Discussion}

According to our experience, the INMEDEA Simulator provides an excellent solution for an interactive casebased learning system that uses virtual patient cases and is based on problem solving (8-10). This simulator helps students acquire practical knowledge that is otherwise rarely taught in medical schools. While classical textbook reading and lectures provide a huge amount of medical facts, students do not necessarily learn what to ask or do in front of a patient. Computerized patient simulation is therefore an excellent method that helps to prepare students to ask appropriate questions and to take appropriate decisions, right from the beginning (11). Furthermore, this virtual patient simulation also offers a practical solution to the frequently encountered problem that only a limited number of real patients are willing to participate in student courses. According to our experiences, the number of students wanting to get hands-on experience examining patients often greatly exceeds the number of patients willing to participate. This problem is even more common in small subspecialties since only a few patients may be hospitalized in the department's wards at a time.

While flight simulation became an obligatory part of pilot training many years ago, patient simulation in medicine remains relatively rare and is often reserved for specific surgical techniques, such as laparoscopy (12) or robotic surgery (13). However, technical procedures (14) and apparently simple situations, including taking a medical history, performing a medical examination, or clarifying the next diagnostic step, can be successfully simulated, as is shown here. The success of the virtual urological cases was demonstrated by both the high acceptance rate of our students (8) and the positive personal impression from medical teachers. Similar experiences have been reported in the literature using other simulators (14) as well as the orthopedic department of the INMEDEA Simulator (10).

Even though this teaching approach has been considered a success by most students, it has often been expressed that neither teaching by a medical tutor nor contact with real patients should be reduced or substituted by any e-learning approach (8). According to students, virtual simulation should be used mainly as a preparatory tool to become familiar with urological diseases in order to be more successful with real patient cases later. Similar statements have been reported in literature (15), which clearly emphasizes that young students prefer live experiences in medical education and desire personalized teaching that cannot be substituted with computerized e-learning. This give us the general impression that case simulations can successfully prepare students for their contact with patients, but should not aim to substitute patient contact completely, especially since many situations in medicine cannot be standardized.

Using the INMEDEA Simulator, students assume the position of the medical doctor in charge, as is described in other studies using simulation exercises (16). This teaches students to decide which medical steps to take next, stimulating their problem-solving skills. In comparison to classical medical teaching, simulation gives students a more active role in learning. Even though virtual patient simulation has several limitations, it also offers many advantages. In contrast to real patients, virtual patients are constantly available and can be examined repeatedly throughout the school year (15). Cases are standardized and equal for all students and can be selected according to the needs of the curriculum. This offers a reliable and comparable learning situation for all students, which is important with respect to medical exams that students are required to take and for reaching defined teaching aims.

As demonstrated in the present work, case creation has become much easier and has been greatly improved by 
the introduction of the authoring tool into the INMEDEA Simulator. Even still, case creation remains a time-consuming and expensive challenge; however, once cases are created, the INMEDEA Simulator offers an excellent opportunity to share cases worldwide. Besides facilitating easier case creation, the authoring tool also allows authors to change or update the case contents and details. This not only allows constant creation of new cases, but also allows the improvement and correction of already existing patient files, contrary to printed media, which must be edited and reprinted before any changes can be made. In this regard, a web-based patient simulation is not a static teaching program like a book, but represents a constantly evolving database that allows quick adaptation to changes in medical content and/or soft- and hardware.

In summary, our experience with the INMEDEA Simulator has demonstrated that teaching using virtual patient cases offers an excellent opportunity for students to improve their practical knowledge on the basis of problem solving. Compared to previous case creation methods, case creation within the current INMEDEA Simulator has become easier due to the integrated authoring tool. The acceptance rates for this teaching approach were high when it was accompanied by classical teaching methods and used under the supervision of medical tutors. With further development of internet and computer technology, it is likely that more virtual patient simulations will become available soon.

\section{Acknowledgments}

None declared.

\section{Financial Disclosure}

Marcus Horstmann received a honorarium from the INMEDEA GmbH company for case creation in 2005 and 2006.

\section{Funding/Support}

None declared.

\section{References}

1. Pinto A, Brunese L, Pinto F, Acampora C, Romano L. E-learning and education in radiology. Eur J Radiol. 2011;78(3):368-71.

2. Pinto A, Selvaggi S, Sicignano G, Vollono E, Iervolino L, Amato F, et al. E-learning tools for education: regulatory aspects, current applications in radiology and future prospects. Radiol Med (Torino). 2008;113(1):144-57.

3. Boulos MN, Maramba I, Wheeler S. Wikis, blogs and podcasts: a new generation of Web-based tools for virtual collaborative clinical practice and education. BMC Med Educ. 2006;6:41.

4. Baldwin A, Webb R, Gainsborough N, Howlett D, Inglis C. Provision of electronic learning resources by UK medical schools for final year students. Med Teach. 2011;33(4):325-7.

5. Ward JP, Gordon J, Field MJ, Lehmann HP. Communication and information technology in medical education. Lancet. 2001;357(9258):792-6.

6. Hurmusiadis V, Rhode K, Schaeffter T, Sherman K. Virtual arthroscopy trainer for minimally invasive surgery. Stud Health Technol Inform. 2011;163:236-8.

7. Hansen MM. Versatile, immersive, creative and dynamic virtual 3-D healthcare learning environments: a review of the literature. J Med Internet Res. [Review]. 2008;10(3):e26.

8. Horstmann M, Renninger M, Hennenlotter J, Horstmann CC, Stenzl A. Blended E-learning in a Web-based virtual hospital: a useful tool for undergraduate education in urology. Educ Health (Abingdon). 2009;22(2):269.

9. Horstmann M, Renninger M, Hennenlotter J, Horstmann CC, Stenzl A. Web-based patient simulation in a virtual department of urology in undergraduate education. Med Teach. [Letter]. 2009;31(9):872-3.

10. Wunschel M, Leichtle U, Wulker N, Kluba T. Using a web-based orthopaedic clinic in the curricular teaching of a German university hospital: analysis of learning effect, student usage and reception. Int J Med Inform. [Research Support, Non-U.S. Gov't]. 2010;79(10):716-21.

11. Cook DA, Erwin PJ, Triola MM. Computerized virtual patients in health professions education: a systematic review and metaanalysis. Acad Med. [Meta-Analysis Research Support, Non-U.S. Gov't Review]. 2010;85(10):1589-602.

12. Ghaderi I, Vaillancourt M, Sroka G, Kaneva PA, Seagull FJ, George I, et al. Performance of simulated laparoscopic incisional hernia repair correlates with operating room performance. Am J Surg. [Research Support, Non-U.S. Gov't]. 2011;201(1):40-5.

13. Seixas-Mikelus SA, Stegemann AP, Kesavadas T, Srimathveeravalli G, Sathyaseelan G, Chandrasekhar R, et al. Content validation of a novel robotic surgical simulator. BJU Int. 2011;107(7):1130-5.

14. Ahmed K, Jawad M, Abboudi M, Gavazzi A, Darzi A, Athanasiou T, et al. Effectiveness of Procedural Simulation in Urology: A Systematic Review. J Urol. 2011;186(1):26-34.

15. Seabra D, Srougi M, Baptista R, Nesrallah LJ, Ortiz V, Sigulem D. Computer aided learning versus standard lecture for undergraduate education in urology. J Urol. 2004;171(3):1220-2.

16. Weller JM. Simulation in undergraduate medical education: bridging the gap between theory and practice. Med Educ. 2004;38(1):32-8. 